\title{
Detection of high coronary risk
}

\author{
GeOFFrey Rose \\ D.M., F.R.C.P., F.F.C.M. \\ Department of Epidemiology and Preventive Medicine, \\ St Mary's Hospital Medical School, London W2 1PG
}

\begin{abstract}
Summary
Signs of early myocardial ischaemia are the most powerful available predictors of myocardial infarction and CHD death. The main primary risk factors are of undiminished importance in such persons, but screening for myocardial ischaemia is not recommended without evidence that intervention at this late stage is effective.

Estimates are presented of the relative importance to individuals of the main primary risk factors. Their importance as population risk factors is different, depending on the product of attributable risk and prevalence: a high risk with low prevalence, as from severe hypercholesterolaemia, is of small importance compared with that arising from the numerous individuals with smaller elevations. Most cases of CHD arise from the common and therefore inconspicuous combination of slight increases in two or three factors in the same individual. Selective and opportunistic risk factor screening is recommended, not a general service.
\end{abstract}

An extension of clinical responsibility in the direction of preventive responsibility is generally accepted as proper, even though it is slow in being applied. When someone with symptomless hypertension is given hypotensive drugs with the object of reducing the risk of stroke, that is preventive medicine. The subject having made no complaint is not strictly a patient: but the clinician accepts a responsibility for him because of the high risk that he will become a patient if nothing is done. This is an analogy for our concern with identifying subjects with a high coronary risk, and the attempt in such persons to prevent the onset of major ischaemia.
Minor ischaemia as a risk factor for major disease

The most effective way to predict myocardial $\vec{\omega}$ infarction and death from coronary heart disease $\stackrel{\circ}{\circ}$ (CHD) is by evidence of early myocardial ischaemia. 8 For example, in various population studies resting 3 . electrocardiography has been used, together with a on simple self-administered questionnaire which iden- 8 tifies angina or history of possible infarction. Figure 8 1 illustrates typical findings: there are indications of $\mathrm{G}$ ischaemia (positive ECG or suggestive symptoms) in $N$ perhaps $160 / 10^{3}$ middle-aged working men, among whom the annual rate of developing major coronary $\overrightarrow{ }$ heart disease proves to be substantially higher than $气$ in those whose examination is negative. A simple screening examination of this kind is able to give $\overrightarrow{0}$ advance warning in almost $50 \%$ of all the men whe will suffer a major coronary event in the next 5 years. Prediction becomes even more powerful if the exanty ination is repeated annually, since these markers of early ischaemia are often transitory: a man may be positive one year, negative the next and the following $\Phi$ year have a fatal infarction. Myocardial infarction $\Rightarrow$ and CHD death are rarely unheralded, but the $\frac{0}{3}$ warnings are often unobserved.

There is an opposite and equally important way of regarding these data. Among men with minor ischaemia the relative risk is high; but in the short or 3 medium term the risk in absolute terms is quite low, and 5 years later $80-90 \%$ of them will be alive and 3 . well. Those who advocate routine screening for myo- $\delta$ cardial ischaemia must consider the numerous false alarms involved. If a man is told that his ECG gives 우 cause for concern, he will never forget it. Anxiety

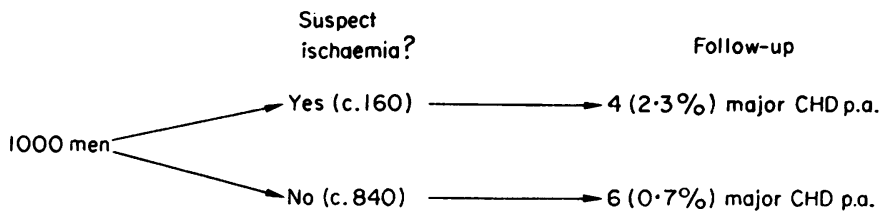

FIG. 1. The prevalence of suspect ischaemia (angina, history of possible infarction or positive ECG) and its relation to the incidence of clinical or fatal CHD over the next 5 years. (Data from a follow-up population survey of men aged 35-39.) 
can be reduced by proper explanation but not abolished, and it can only be justified by good evidence that the inevitable harm can be offset by a corresponding hope of benefit. It is only proper to uncover a high risk state if risk is known to be reversible. At the stage when myocardial injury has occurred, it must be asked whether the process may not have advanced too far to be effectively slowed.

There is recent evidence showing that even in those individuals who have progressed to this stage of early ischaemic injury, the main primary risk factors (smoking and the levels of blood pressure and cholesterol) are still major predictors of the outcome. Thus each of these factors seems to increase the risk of CHD death at least as much in men with, for example, ischaemic $S-T / T$ changes as in the rest. This is certainly encouraging for the possibility of prevention being effective even at this late stage, but it still needs a controlled trial to discover the effect of modifying risk factors in this important group. Until this has been done it would not be right to press screening for early myocardial ischaemia. However, whenever such evidence of ischaemia happens to come to a doctor's notice, it should underline the urgency of controlling the primary risk factors.

\section{Primary risk factors}

Of the primary risk factors age is the strongest predictor: CHD incidence doubles in middle-aged people every 7 or 8 years. On the other hand, the older the person being dealt with, the less the urgency of preventive action, because the tragedy of an early death is felt more strongly as compared with death in old age.

Male sex is also a powerful identifier of risk, although the mortality rates for CHD in the two sexes converge steadily throughout life and eventually they almost meet. It would nevertheless be well to consider that if CHD did not occur at all in men, then it would be reckoned a terrible scourge among women. Women are not really at low risk: their problem is simply overshadowed by the extremely high risk in men.

There are three key factors for identifying persons with a special need for preventive advice, and these happen also to be the three factors on whose modification it is desirable to concentrate: they are smoking, blood pressure and blood cholesterol. Table 1 shows their relative power as risk factors in middleaged men, taking as unity the risk in the happy individual who does not smoke and who has low levels of blood pressure and cholesterol. Risk is approximately doubled by either fifteen cigarettes daily or by $20 \mathrm{~mm}$ elevation of diastolic pressure or by a cholesterol level of $250 \mathrm{mg} / \mathrm{dl}$. Each of these is roughly equivalent in its effect on risk.

Clinical interest in identifying high risk has been
TABLE 1. Equivalent levels of the three main risk factors in middle-aged men

\begin{tabular}{cccc}
\hline & \multicolumn{2}{c}{ Equivalent level of risk factor } \\
\cline { 2 - 4 } $\begin{array}{c}\text { Relative risk } \\
\text { of CHD }\end{array}$ & Cigs/day & BP (diast) & $\begin{array}{c}\text { Serum } \\
\text { cholesterol } \\
(\mathrm{mg} / \mathrm{dl})\end{array}$ \\
\hline 1 & 0 & 80 & 180 \\
2 & 15 & 100 & 250 \\
5 & 40 & 120 & 350 \\
\hline
\end{tabular}

concentrated mainly on the high tail of the frequency distribution for each particular factor-the patients with clinically significant hypertension or with severe hypercholesterolaemia. Of course such individuals are indeed at high risk of CHD. But such cases must be seen in the perspective of the main problem, which is the community control of this disease. For example, familial hyperbetalipoproteinaemia is indeed very bad for those who have it, with more than $50 \%$ of affected males dying from premature CHD; but from a community point of view it represents the barest fringe of the coronary heart disease problem, accounting for no more than $1 \%$ of all CHD deaths. A strategy of CHD control based on the few individuals with high values for single factors is not going to contribute much to the control of disease in the population as a whole.

This can be illustrated by some calculations which can be made from epidemiological data, taking men aged 55-64 in the Framingham study as an example. Figure 2 shows first a graph (the broken line) relating CHD mortality to cholesterol level at entry to the study. The base of this scale is set at the level of risk for men in the lowest cholesterol class, so that the height above the base at each level of cholesterol represents the excess or attributable risk. Concern is commonly concentrated on individuals in the upper cholesterol classes, where the risk of CHD is admittedly high. However, a strategy of community control must consider not only the risk in any particular group of individuals, but also the number of such persons in the population. These prevalence values are shown in the histogram (Fig. 2). By simple cross-multiplication of the prevalence rates with the corresponding individual attributable risks one may calculate 'population attributable risk'. The results, which are shown as numbers at the top of each bar in the histogram, represent the actual numbers of CHD deaths in this population group which are statistically attributable to each particular level of cholesterol elevation. It is seen that out of the total of fourteen CHD deaths $/ 10^{3} / 10$ years which are statistically attributable to cholesterol elevation, only one arises in those levels $(>310 \mathrm{mg} / \mathrm{dl}, 8 \mathrm{mmol} / \mathrm{l})$ that are likely to arouse clinical concern, whereas seven $(50 \%)$ arise at levels below $250 \mathrm{mg} / \mathrm{dl}(6.5 \mathrm{mmol} / \mathrm{l})$ : 


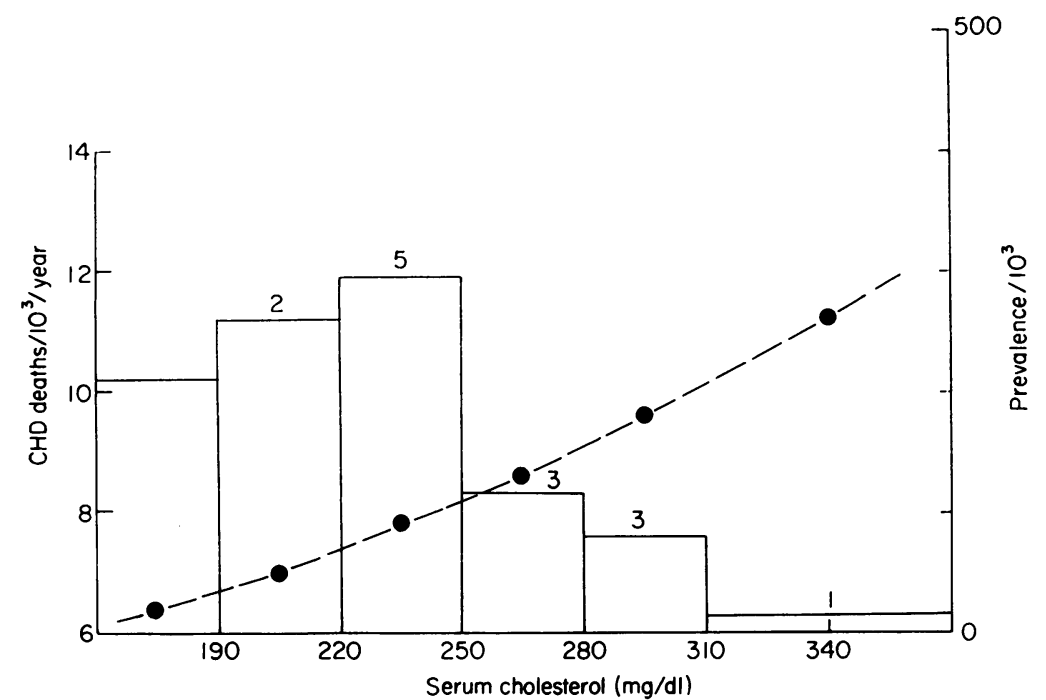

FIG. 2. The relation between serum cholesterol level and attributable CHD mortality (broken line), the prevalence rates for various cholesterol levels (histogram), and the number of attributable deaths arising at these levels in a population of 1000 men aged 55-64 during a 10-year period. (Calculated from data of Shurtleff, 1970.)

TABLE 2. Population attributable risk associated with various factors, and the level of each factor below which two-thirds (approx.) of the attributable risk arises. (Calculated from data of Shurtleff, 1970)

\begin{tabular}{lcl}
\hline \multicolumn{1}{c}{ Factor } & $\begin{array}{c}\text { Population } \\
\text { attributable } \\
\text { risk (\%) }\end{array}$ & $\begin{array}{c}\text { Two-thirds (approx.) } \\
\text { of risk } \\
\text { arises below }\end{array}$ \\
\hline Systolic BP & 51 & $170 \mathrm{~mm}$ \\
Serum urate & 26 & $6 \mathrm{mg} / \mathrm{dl}(360 \mu \mathrm{mol} / \mathrm{l})$ \\
Relative weight & 24 & $15 \%$ overweight \\
Serum cholesterol & 18 & $280 \mathrm{mg} / \mathrm{dl}(7 \cdot 2 \mathrm{mmol} / \mathrm{l})$ \\
Cigarettes & 18 & $20 \mathrm{cigs} / \mathrm{day}$ \\
Blood sugar (casual) & 11 & $120 \mathrm{mg} / \mathrm{dl}(6.7 \mathrm{mmol} / \mathrm{l})$ \\
\hline
\end{tabular}

many individuals at slight risk contribute far more to the community burden than a few individuals at high risk.

Table 2 shows some results for similar calculations of population attributable risk for the other main risk factors, again using data for Framingham men aged 55-64. The figures in the first column show the percentage of all CHD deaths which are statistically attributable to the factor in question. (The total adds up to more than $100 \%$ because the factors are intercorrelated.) These represent the proportion by which CHD mortality would fall in this population if one could abolish the whole of the risk associated statistically with that factor, that is, the theoretical maximum contribution from a wholly effective control programme; and they are calculated from products of individual risk and prevalence rates. These results permit comparison of the relative importance of the various risk factors in this particular population; they emphasize the dominating importance of blood pressure as a population risk factor for CHD.

The final column of Table 2 shows the cut-off point in the distribution of each risk factor such that about one-third of attributable cases arise above that level and two-thirds below it. In each instance it is seen that only a small community benefit can be expected if preventive action is restricted to individuals who lie in the upper extreme of the distribution for a single risk factor.

\section{Multifactorial risk}

There is substantial though not complete independence between the main risk factors, so that the risk for a man with two factors is not far short of the product of the two separate relative risks. This means that the absolute risk associated with a particular factor is not the same for everyone but depends on the context in which it occurs: it is thus essential to assess an individual's profile as a whole. An example of this is illustrated in Table 3, which is derived from Framingham data for 50-year-old men: it shows that the excess CHD risk associated with cigarette smoking is more than three times greater in the presence of higher levels of blood pressure and cholesterol. A similar effect holds for other combinations of the main risk factors.

In an earlier paper in this symposium Professor Blackburn (1976) has shown the necessity of a multifactorial approach to risk identification, following 
TABLE 3. Contrasts in CHD risk of smoking according to presence or absence of other risk factors. (Calculated from data for 50-year-old Framingham men: Gordon, Sorlie and Kannel, 1971)

\begin{tabular}{|c|c|c|}
\hline & \multicolumn{2}{|c|}{10 -year risk of CHD } \\
\hline & $\begin{array}{c}\text { Blood pressure } \\
\text { and cholesterol } \\
\text { low }\end{array}$ & $\begin{array}{c}\text { Blood pressure } \\
\text { and cholesterol } \\
\text { high }\end{array}$ \\
\hline $\begin{array}{l}\text { Non-smokers } \\
\text { Cigarette smokers } \\
\text { Excess in smokers }\end{array}$ & $\begin{array}{r}4 \cdot 2 \% \\
6 \cdot 5 \% \\
+2 \cdot 3 \%\end{array}$ & $\begin{array}{r}17 \cdot 5 \% \\
26 \cdot 0 \% \\
+8 \cdot 5 \%\end{array}$ \\
\hline
\end{tabular}

from the fact that high risk is in most cases due to combinations of several slightly elevated factors rather than to one factor that is much elevated. The seriousness of a plasma cholesterol of, say, 280 $\mathrm{mg} / \mathrm{dl}$ cannot be assessed unless we know the individual's whole risk factor profile: it is great in a sedentary smoker with slight hypertension, but small in the absence of these other adverse factors. Similarly the management of high-risk subjects must be multifactorial. Smoking in a mild hypertensive is far more serious than in a normotensive, and to leave it untreated may be as serious as to neglect the blood pressure. Whenever we see a patient known to be high risk (a hypertensive or a diabetic, for instance) this identifies someone in whom all the other risk factors take on a particular importance.

\section{Screening to identify high CHD risk}

In the course of ordinary clinical examination we are constantly 'screening' the population. We examine all systems whether we are suspicious of them or not, and in this way a lot of unsuspected risk factor information is uncovered from family history, or slight elevation of blood pressure, or blood lipids (from an SMA 12, for example), or from ECG signs of early myocardial ischaemia. The information is there in front of us and it should be used and not ignored. Particularly we should look out for those combinations of slight elevations of several factors which together can add up to a risk as high as that of the patient with gross hyperlipidaemia or hypertension.
Planned screening, conducted for the express purpose of identifying high risk individuals, is a more difficult issue. The author suggests that it is well to screen, where feasible and acceptable, the firstdegree relatives of young patients with myocardial infarction, hypertension or major hypercholesterolaemia. He does not think we have yet reached the point of advocating general risk factor screening outside these restricted groups. The situation is different if an individual himself takes the initiative by going to his doctor and saying, 'I have read about the things I am supposed to do to avoid heart attack, but I want to know how important it all is in my particular case: how much need is there for me to change my way of life?' That seems a reasonable request, and it is thought that doctors should provide risk assessment on demand in such cases.

Where it is not requested in this way, it is suggested that general screening for risk factors should not be undertaken. Its main function would be to enhance motivation, providing a stick with which to beat the recalcitrant. It does not need screening to identify smoking, physical inactivity or overweight; if doctors do their job, most adults can have blood pressure measurement in the course of normal clinical contacts; and considering the fat content of the modern British diet, it does not require a blood test to conclude that nearly everyone's blood cholesterol level is higher than it ought to be. Before instituting new services we should first do more for the many high risk individuals whose need is already obvious.

\section{References}

Blackburn, H. (1976) Concepts and controversies about the prevention of coronary heart disease. Postgraduate Medical Journal, 52, 417.

Gordon, T., Sorlie, P. \& KANNEL, W.B. (1971) The Framingham Study. Section 27. Coronary heart disease, atherothrombotic brain infarction, intermittent claudication-a multivariate analysis of some factors related to their incidence: Framingham Study, 16-year follow-up. U.S. Govt. Printing Office, Washington, D.C., U.S.A.

Shurtleff, D. (1970) The Framingham Study. Section 26. Some characteristics related to the incidence of cardiovascular disease and death: Framingham Study, 16-year follow-up. U.S. Govt. Printing Office, Washington, D.C., U.S.A. 